University of Nebraska - Lincoln

DigitalCommons@University of Nebraska - Lincoln

2-4-2009

\title{
Changes in the Adsorbate Dipole Layer with Changing d-Filling of the Metal (II) (Co, Ni, Cu) Phthalocyanines on $\mathrm{Au}(111)$
}

Jie Xiao

University of Nebraska-Lincoln, jie.xiao@helmholtz-berlin.de

Peter A. Dowben

University of Nebraska-Lincoln, pdowben@unl.edu

Follow this and additional works at: https://digitalcommons.unl.edu/physicsdowben

Part of the Physics Commons

Xiao, Jie and Dowben, Peter A., "Changes in the Adsorbate Dipole Layer with Changing d-Filling of the Metal (II) (Co, Ni, Cu) Phthalocyanines on Au(111)" (2009). Peter Dowben Publications. 224.

https://digitalcommons.unl.edu/physicsdowben/224

This Article is brought to you for free and open access by the Research Papers in Physics and Astronomy at DigitalCommons@University of Nebraska - Lincoln. It has been accepted for inclusion in Peter Dowben Publications by an authorized administrator of DigitalCommons@University of Nebraska - Lincoln. 


\title{
Changes in the adsorbate dipole layer with changing d-filling of the metal (II) $(\mathrm{Co}, \mathrm{Ni}, \mathrm{Cu})$ phthalocyanines on $\mathrm{Au}(111)$
}

\author{
Jie Xiao and Peter A. Dowben
}

Department of Physics and Astronomy, Nebraska Center for Materials and Nanoscience, University of Nebraska-Lincoln, Lincoln, NE 68588-0111, USA

Corresponding author-P. A. Dowben, email pdowben@unl.edu

\begin{abstract}
In combined photoemission and inverse photoemission spectroscopy studies, we observe changes in the metal phthalocyanine molecular orbital offsets with respect to the conducting gold substrate Fermi level, with the changing d-electron filling of the metal (II) $(\mathrm{Co}, \mathrm{Ni}, \mathrm{Cu})$ phthalocyanines. The implication is that the interfacial dipole layer depends upon the choice of metal (Co, Ni, $\mathrm{Cu}$ ) centers within the metal (II) phthalocyanines adsorbed on $\mathrm{Au}(111)$.
\end{abstract}

For many large molecule adlayers, including a number of organic and metal-organic species, the energy level alignment of the adsorbate with respect to a conducting substrate Fermi level or adjacent layer chemical potential is dependent on the interfacial electronic structure and interfacial dipole layer, as has been demonstrated for a number of molecular species [1-3], including the metal (II) phthalocyanines (MPc) $[4,5]$. The metal (II) phthalocyanines offer a family of compounds where the d-electron filling of the metal center atom can be systematically altered, without changing the apparent preferential bonding orientation on $\mathrm{Au}(111)[6,7]$. This persistent orientation with the molecular plane parallel with the substrate for very thin films of adsorbed MPc species $[6,7]$ is unlike the case for the macrocyclic metal center tetraazaannulenes (TMTAA) [8]. If the interface dipole formation matters, then metal (II) phthalocyanines adsorbed on $\mathrm{Au}(111)$ will be expected to show different molecular orbital alignments with different metal substitutions since metal centers have different $3 \mathrm{~d}$ orbital configurations $\left(\mathrm{Co}^{2+} \mathrm{d}^{7}, \mathrm{Ni}^{2+} \mathrm{d}^{8}\right.$ and $\left.\mathrm{Cu}^{2+} \mathrm{d}^{9}\right)$. In other words, we expect that the interfacial dipole will alter the placement of the molecular orbitals with respect to the substrate Fermi level in a similar manner that electron filling and depletion alters the placement of the molecular orbitals, but not the ordering, of an organic semiconductor [9].

While there are some indications that the $3 \mathrm{~d}$ transition metal does affect the molecular orbital alignment from scanning tunneling microscopy (STM) studies [6, 7], no systematic combined photoemission and inverse photoemission studies have been undertaken to experimentally elucidate the molecular band offsets for a family of metal (II) phthalocyanines. Such combined photoemission studies are one of the best methods for identifying the affects of initial state electron filling and depletion on an organic semiconductor molecular orbital placement [9] and distinguishing such effects from final state effects like charging and changes in the electron or hole screening in a final state spectroscopy like photoemission [10, 11]. Model theoretical calculations suggest that most of the molecular orbitals, including the highest occupied molecular orbital (HOMO) and lowest unoccupied molecular orbital (LUMO) are dominated by the phthalocyanine $(\mathrm{Pc})$ ligands [12-15]. The exception to this overly general statement would be cobalt phthalocyanine (CoPc) where the cobalt $3 \mathrm{~d}_{z}^{2}$ weighted molecular orbital (with the $z$-axis defined here to be along the surface normal) is expected to lie less than $1 \mathrm{eV}$ below the highest occupied molecular orbital (HOMO) of the ligand (i.e. no more than $1 \mathrm{eV}$ greater binding energy relative to the $\mathrm{HOMO})[6,16]$. For cobalt phthalocyanine $(\mathrm{CoPc})$ the cobalt $3 \mathrm{~d}_{z}^{2}$ weighted molecular orbital may be responsible for the appearance of the central "bright spot" in scanning tunneling microscopy (STM) images of CoPc $[6,16]$. 


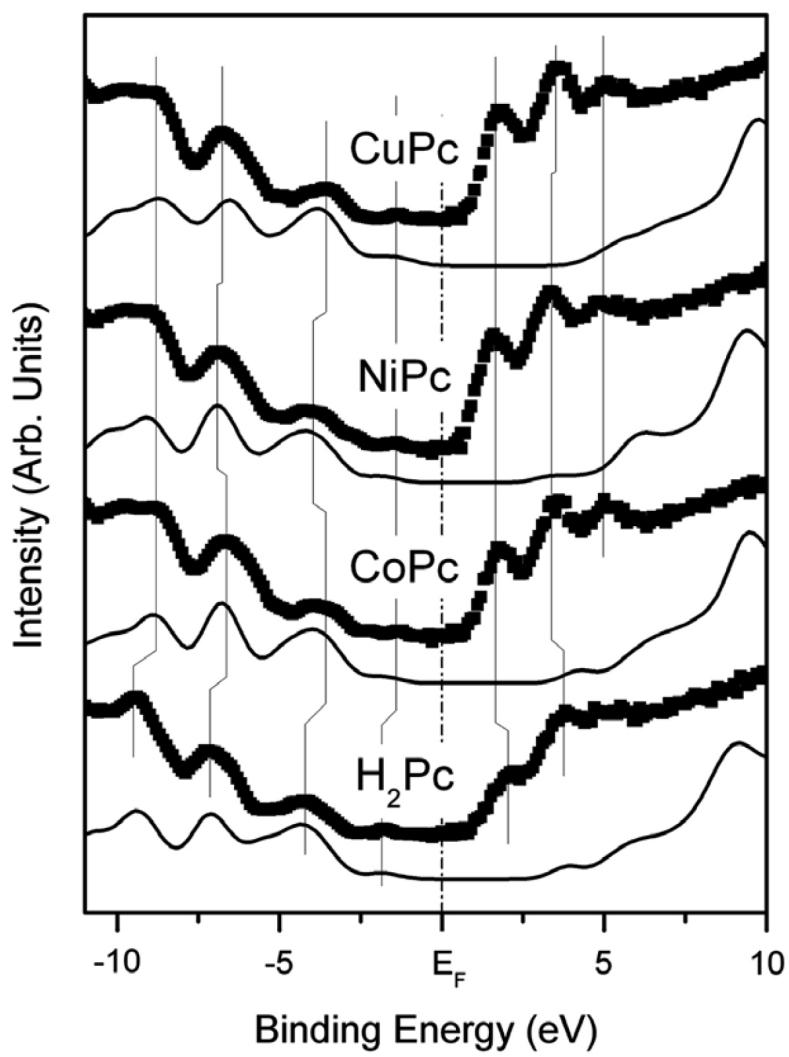

Figure 1. The photoemission and inverse photoemission spectra (thick lines) of molecular multilayer $\mathrm{CoPc}, \mathrm{NiPc}, \mathrm{CuPc}$ and $\mathrm{H}_{2} \mathrm{Pc}$ thick films adsorbed on $\mathrm{Au}(111)$ substrate at room temperature. Model calculations of the single molecule density of states, using a semi-empirical approach uncorrected for matrix elements and final state effects [3, 5, $8,9]$, are shown for comparison as a thin line for each species. Vertical bars roughly indicate the approximate molecular orbital (band) offsets between metal phthalocyanines and $\mathrm{H}_{2} \mathrm{Pc}$.

Combined photoemission and inverse photoemission spectra were taken from different coverage of metal $\left(\mathrm{Co}^{2+}\right.$, $\mathrm{Ni}^{2+}, \mathrm{Cu}^{2+}$ ) phthalocyanines on $\mathrm{Au}(111)$ substrate, nominally from one monolayer to the thicker multilayer films. In the case of thicker molecular films studied, thicknesses far greater than the mean free path of the photoelectrons emitted from the gold substrate by He I radiation $(21.2 \mathrm{eV}$ photon energy) were chosen, but still at a film thickness where the molecular plane of the metal phthalocyanine remained largely parallel with the interface. The MPc films were grown on $\mathrm{Au}(111)$ substrates by evaporation of the commercially available $\mathrm{CoPc}, \mathrm{NiPc}$ and $\mathrm{CuPc}$ powders, in a preparation chamber vacuum continuous with the ultrahigh vacuum (UHV) chamber equipped with both photoemission and inverse photoemission spectroscopies $[5,9,17$, 18]. The clean gold surface was characterized by photoemission and inverse photoemission spectra, and used to establish the substrate Fermi level which is the reference energy for all spectra reported here. The photoemission spectra were taken with a hemispherical electron energy analyzer using He I radiation of $21.2 \mathrm{eV}$, with the photoelectrons collected along the surface normal. The inverse photoemission spectra were taken in the same UHV chamber using a Geiger-Müller detector with the electrons incident along the surface normal.
The photoemission spectroscopy studies, as predicted by theory, illustrate the very similar electronic structure of the various MPc adsorbed species for thicker molecular films [19-24]. Indeed, the combined occupied and unoccupied electronic structures of metal $(\mathrm{Co}, \mathrm{Ni}, \mathrm{Cu})$ phthalocyanine "thick" films on $\mathrm{Au}(111)$ (Figure 1) from UPS and IPES provide the expected agreement with prior experimental studies and theory [5, 19-24], although there is an observed decrease, compared to our theoretical expectations, in the single molecule HOMO-LUMO gap due to expected solid state effects. Such expected solid state effects include intermolecular interactions [25] that would tend to lead to a decrease in the HOMO-LUMO gap. In spite of the difference in the HOMO-LUMO gap seen in experiment, from the expectations of model ground state calculations, the qualitative agreement with the combined photoemission and inverse photoemission results tend to confirm that most of the observed photoemission features are from the occupied molecular phthalocyanine $(\mathrm{Pc})$ ligand.

There are a number of complications one needs to consider in comparing the adsorbed metal phthalocyanine films on $\mathrm{Au}(111)$. There are indications that for thick MPc films, the plane of the molecule tends to tilt up, adopting molecular orientations that are not coplanar with the substrate surface [21], an undesirable molecular orientation (for the purposes of the comparison discussed herein) that we have made every attempt to avoid in our studies here. Furthermore, the actual molecular film thickness may not always be uniform, as in the case of CoPc which exhibits StranskiKrastanov growth on some substrates [22]. Fortunately, the interfacial dipoles caused by charge transfer between adsorbed metal phthalocyanines (MPc) and the Au(111) substrate have little influence on the spectra of these thicker MPc films as the interface and the immediate interface molecular layers are buried far below from the topmost surface of the thicker molecular films and are thus at distance larger than the photoelectron mean free path: the Au substrate features are not seen to contribute to any of the spectra of Figure 1. Thus the strong similarities seen in comparing the various metal phthalocyanines, are expected for the thicker molecular films.

The comparison of the combined photoemission and inverse photoemission spectra for the non-metal phthalocyanine and the metal $\left(\mathrm{Co}^{2+}, \mathrm{Ni}^{2+}, \mathrm{Cu}^{2+}\right)$ phthalocyanine molecular films (Figure 1) indicates that the $3 \mathrm{~d}$ orbital contributions of the metal centers to the overall molecular spectra lead to either an additional final state screening channel or an initial state decrease in the highest occupied molecular orbital (HOMO) to lowest unoccupied molecular orbital (LUMO) gap.

Because the model calculations suggest that the $3 \mathrm{~d}$ orbital contributions of the metal centers to the overall molecular orbitals are far smaller than is in fact observed in Figure 1, we are forced to conclude that $3 \mathrm{~d}$ orbital contributions to the molecular orbitals affects both the initial and final states in photoemission and inverse photoemission. The transition metal centers of metal phthalocyanines bring an extra $3 \mathrm{~d}$ orbital interaction with molecular ligand and adjacent adsorbate molecules and provide extra final state screening channels for the photoemission and inverse 


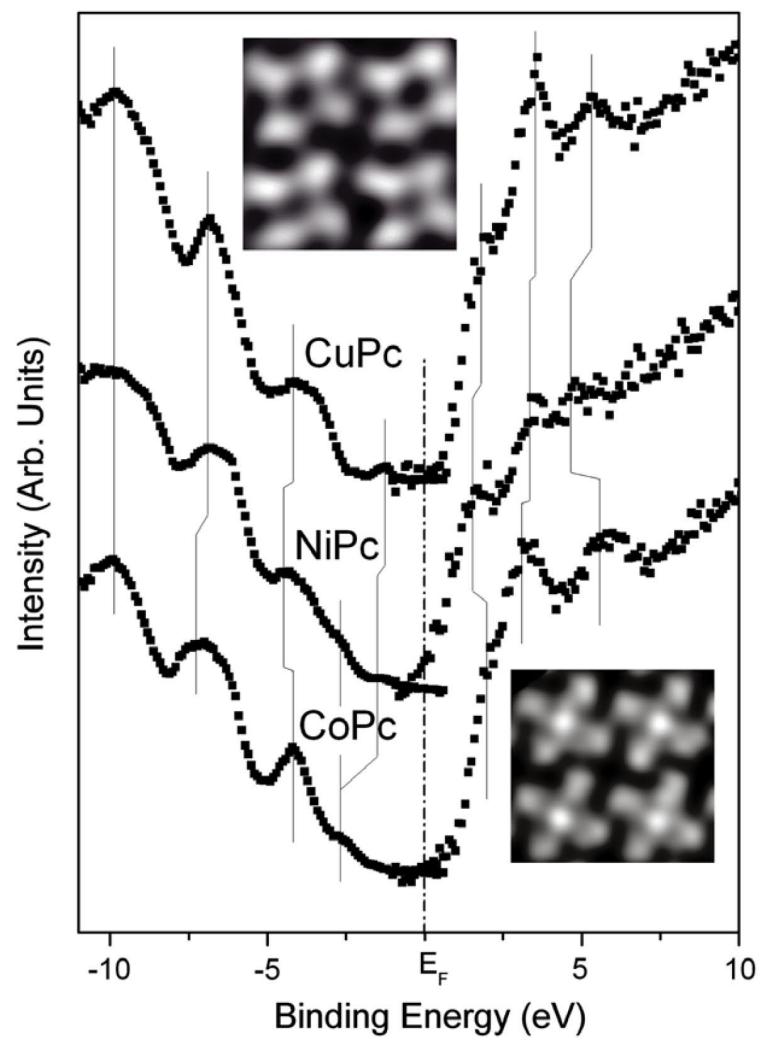

Figure 2. Photoemission and inverse photoemission spectra of $\mathrm{CoPc}$, $\mathrm{NiPc}$ and $\mathrm{CuPc}$ thin films adsorbed on $\mathrm{Au}(111)$ substrate at room temperature. Fermi level is referenced to Au substrate. Molecular orbital offsets among $\mathrm{CoPc}$, NiPc and $\mathrm{CuPc}$ are marked by vertical bars. The STM insets for adsorbed CuPc (at top) and CoPc (at bottom) are reproduced from [6], with permission.

photoemission processes. The addition of the metal center results in the appearance of a smaller HOMO-LUMO gap for the intra-molecular exited final states [11] than is observed in the photoemission and inverse photoemission from the metal absent center molecule $\mathrm{H}_{2} \mathrm{Pc}$, as seen in Figure 1. A significant displacement of all the molecular orbitals (collectively) for one of the phthalocyanine adsorbate species studied, generally to greater or lesser binding energies, is not observed in these thicker molecular films (Figure 1), so a pure initial state chemical shift is unlikely. Model calculations (both semi-empirical PM3 and DFT) by ourselves (Figure 1) and others indicate that all three species have similar placements of the chemical potential, as prior theoretical calculations also note [12-16]. This is illustrated in Figure 1 where the model single molecule density of states (PM3) for each species has been shifted by some $6.0 \mathrm{eV}$ (a value of about expected work function) to align the calculated orbital energies with photoemission experiments. Thus the similar alignment of molecular orbitals in photoemission and inverse photoemission for the thicker molecular films is as expected.

To study the interfacial electronic structures and interaction between the metal $(\mathrm{Co}, \mathrm{Ni}, \mathrm{Cu})$ phthalocyanines and the $\mathrm{Au}(111)$ substrate, photoemission and inverse photoemission spectra were taken from "thin" molecular films (Figure 2), for a film thickness between 1 and 2 molecular monolayers. For such MPc films, all the molecules lie flat on the $\mathrm{Au}(111)$ surface with the molecular ligand copla-

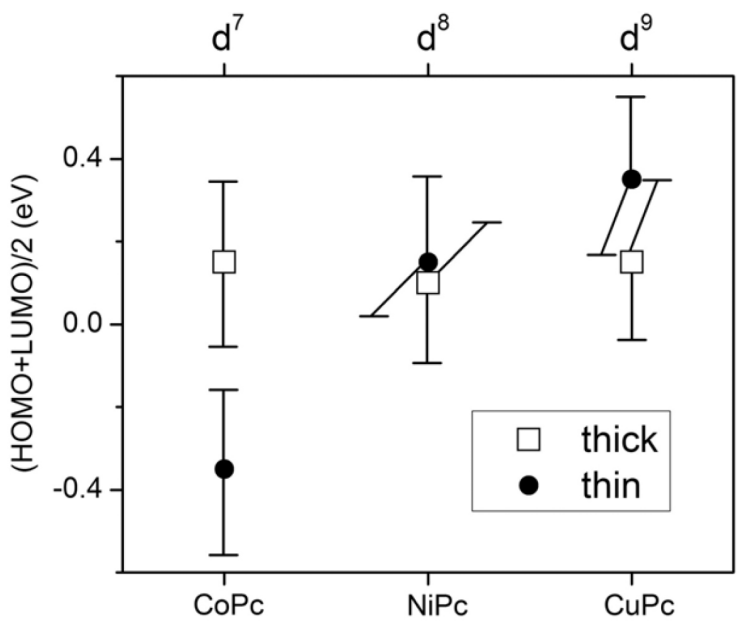

Figure 3. A comparison of the molecular chemical potential (HOMOLUMO gap center) with respect to the Fermi level between the thin and thick MPc films. Filled circles represent the thin films of MPc, empty squares represent thick films.

nar with the substrate surface. For these thinner molecular films, the combined photoemission and inverse photoemission spectra generally show different molecular orbital alignments with respect to the substrate Fermi level compared to the thicker films. The molecular orbital alignments with respect to the substrate Fermi level also systematically vary for each metal $(\mathrm{Co}, \mathrm{Ni}, \mathrm{Cu})$ phthalocyanine according to the $3 \mathrm{~d}$ metal occupancy. These shifts in the molecular orbital alignments with respect to the substrate Fermi level are indicated by vertical bars in Figure 2. The Fermi level moves towards the lowest partially occupied molecular orbital for CoPc and towards the highest partially occupied molecular orbital for $\mathrm{CuPc}$, while for the thinner NiPc molecular films on $\mathrm{Au}(111)$, the molecular orbital features are almost at the same position relative to the substrate Fermi level, as in the spectra of "thicker" NiPc films.

The substrate interactions do not simply alter the molecular orbital alignment relative to the substrate Fermi level, but also affect the placement of the molecular orbitals in other ways, as observed in photoemission and inverse photoemission. The highest occupied molecular orbital (HOMO) to lowest unoccupied molecular orbital (LUMO) gap increases up a little for all three metal $(\mathrm{Co}, \mathrm{Ni}, \mathrm{Cu})$ phthalocyanines, when the molecular films are very thin. This effect is quite large for CoPc and indeed for CoPc, both the highest occupied and lowest lying unoccupied molecular orbitals are strongly perturbed in photoemission and inverse photoemission respectively, as indicated in Figure 2. The strong perturbation of the CoPc molecular orbitals, for the thinner $\mathrm{CoPc}$ films on $\mathrm{Au}(111)$, supports the contention of Lu et al [6] that CoPc is more strongly adsorbed on $\mathrm{Au}(111)$ than $\mathrm{CuPc}$.

In Figure 3, we have plotted the center of the experimentally determined adsorbate HOMO-LUMO gap, obtained from the combined photoemission and inverse photoemission studies, with respect to the substrate Fermi level for both thin and thick films. As noted above, the molecular orbital alignment is little different for the three metal $(\mathrm{Co}, \mathrm{Ni}, \mathrm{Cu})$ phthalocyanines, when the molecular films are rather thick. 
In the thinner molecular films, the large shifts in the molecular orbital alignment suggest charge transfer between the $\mathrm{CuPc}$ and $\mathrm{CoPc}$ adsorbate species and the substrate. In the model of orbital filling and depletion [9], the summary plots of the changes in the molecular orbital alignment for the thinner molecular films in Figure 3 indicate that there is electron charge transfer from Au substrate to CoPc. For $\mathrm{CuPc}$ on $\mathrm{Au}(111)$, the electron charge transfer is in the opposite direction: from the adsorbate to the substrate. For $\mathrm{NiPc}$, the small shift in the molecular orbital alignment suggests little charge transfer could be observed from the photoemission and inverse photoemission spectra and there is little change in the adsorbate molecular orbital charge occupancy, even for the thinner molecular films on $\mathrm{Au}(111)$. In general, such electron filling and depletion of the molecular orbitals of the adsorbate species, as observed here at the $\mathrm{Au}(111)$ molecular film interface, results in the formation of different interfacial dipoles. Electron filling tends to move the Fermi level position towards the conduction band edge and electron depletion of the molecular orbitals would tend to move the molecular orbital alignment so that the Fermi level is closer to the valence band edge [9]. Band bending at the interface, as occurs with semiconductors, will be unlikely in these systems because of low charge mobility along the surface normal inside the metal $(\mathrm{Co}, \mathrm{Ni}, \mathrm{Cu})$ phthalocyanine thin film and because of the short extinction length of the molecular orbital wavefunctions outside each individual molecule [26, 27].

Charge depletion from $\mathrm{CuPc}$ would tend to deplete the $\mathrm{Cu} 3 \mathrm{~d}_{z}^{2}$ orbital, through the ligand, and lead to an increase of the orbital binding energy, if the ligand to metal bond strength remains largely preserved (again with the $z$-axis defined along the surface normal). Charge depletion from $\mathrm{Cu} 3 \mathrm{~d}^{2}$ orbital means that the occupied $\mathrm{Cu} 3 \mathrm{~d}$ states will be harder to observe in scanning tunneling microscopy. Because the occupied $\mathrm{Cu} 3 \mathrm{~d}_{x z}$ and $3 \mathrm{~d}_{y z}$ are not fully symmetric states, i.e. they are elements in the $\mathrm{E}$ irreducible representation, not the fully symmetric $\mathrm{A}_{1}$ irreducible representation in $\mathrm{C}_{4 \mathrm{v}}$, the tunneling matrix elements will be smaller than expected for the $\mathrm{Cu} 3 \mathrm{~d}_{z}^{2}$ orbital [28-33]. Tunneling matrix elements that apply to scanning tunneling microscopy tend to favor the electron states that are fully symmetric: the $A_{1}$ irreducible representation in $\mathrm{C}_{4 \mathrm{v}}$ containing states with $\mathrm{s}, \mathrm{p}_{z}$ and $\mathrm{d}_{z}^{2}$ contributions. While the highest occupied molecular orbitals of $\mathrm{CuPc}$ will be placed closer to the Fermi level at the molecule/ $\mathrm{Au}(111)$ interface than is the case for either NiPc or $\mathrm{CoPc}$, the $\mathrm{Cu} 3 \mathrm{~d}_{z}^{2}$ and s weighted molecular orbitals, with the large tunneling matrix elements values, remain placed well away from the Fermi level, thus a change in the symmetric state occupancy $\left(\mathrm{A}_{1}\right.$ irreducible representation in $\mathrm{C}_{4 \mathrm{v}}$ ) will have a profound effect on the how well the $\mathrm{Cu}$ metal center atom is imaged. The depletion of the $\mathrm{Cu}$ $3 \mathrm{~d}_{z}^{2}$ orbital occupancy through the ligand, due to interface bonding, means that the $\mathrm{Cu}$ center atom will be harder to image for $\mathrm{CuPc}$ adsorbed on $\mathrm{Au}(111)$ in scanning tunneling microscopy than the metal center of the other phthalocyanine. While there is difficulty in imaging the metal center for $\mathrm{CuPc}$, the ligand for $\mathrm{CuPc}$ should be brighter as is indeed observed [6] and illustrated in Figure 2 as an inset. On the other hand, charge donation to the adsorbed CoPc will tend to be through frontier orbitals of the $\mathrm{Au}(111)$ substrate: $6 \mathrm{~s}$ and $5 \mathrm{~d}_{z}^{2}$ with spectral weight located near the Fermi level. Such charge contributions will enhance the Co $3 \mathrm{~d}_{z}^{2}$ orbitals which means that tunneling at the metal center position for CoPc is highly likely, if substrate contributions are included for low bias voltages (as occurs with alkali metal adsorption on metal surfaces [34]). This increase in tunneling current at the metal center position for $\mathrm{CoPc}$ on $\mathrm{Au}(111)$ has also been observed [6], and again illustrated in Figure 2 as an inset.

In conclusion, we have demonstrated that molecular orbital filling and depletion of the adsorbed molecular metal (Co, Ni, Cu) phthalocyanines at the $\mathrm{Au}(111)$ interface results in different energy level alignments for metal phthalocyanines with different metal centers that scale with the metal d-filling.

This research was supported by the National Science Foundation through grant number CHE-0415421 and CHE0650453. The authors are grateful to K. W. Hipps for helpful conversations and permission to reproduce the STM images in Figure 2.

\section{References}

[1] Ishii H, Sugiyama K, Ito E, and Seki K 1999 Adv. Mater. 11 605

[2] Zhu X Y 2004 Surf. Sci. Rep. 561

[3] Balaz S, Caruso A N, Platt N P, Dimov D I, Boag N M, Brand J I, Losovyj Ya B, and Dowben P A 2007 J. Phys. Chem. B 111 7009

[4] Kera S, Yabuuchi Y, Yamane H, Setoyama H, Okudaira K K, and Ueno N 2004 Phys. Rev. B 70085304

[5] Xiao J, Sokolov A, and Dowben P A 2007 Appl. Phys. Lett. 90242907

[6] Lu X, Hipps K W, Wang X D, and Mazur U 1996 J. Am. Chem. Soc. 1187197

[7] Lu X and Hipps K W 1997 J. Phys. Chem. B 1015391

[8] Liu J, Xiao J, Choi S-B, Jeppson P, Jarabek L, Losovyj Ya B, Caruso A N, and Dowben P A 2006 J. Phys. Chem. B 110 26180

[9] Xu B, Choi J, Caruso A N, and Dowben P A 2002 Appl. Phys. Lett. 804342

[10] Ortega J E, Himpsel F J, Li D, and Dowben P A 1994 Solid State Commun. 91807

[11] Dowben P A 2000 Surf. Sci. Rep. 40151

[12] Rosa A and Baerends E J 1994 Inorg. Chem. 33584

[13] Reynolds P A and Figgis B N 1991 Inorg. Chem. 302294

[14] Scrocco M, Ercolani C, and Paoletti A M 1993 J. Electron Spectrosc. Relat. Phenom. 63155

[15] Suzuki T, Kurahashi M, Ju X, and Yamauchi Y 2002 J. Phys. Chem. B 10611553

[16] Barlow D E, Scudiero L, and Hipps K W 2004 Langmuir 20 4413

[17] McIlroy D N, Waldfried C, McAvoy T, Choi J, Dowben P A, and Heskett D 1997 Chem. Phys. Lett. 264168

[18] Choi J, Dowben P A, Borca C N, Adenwalla S, Bune A V, Ducharme S, Fridkin V M, Palto S P, and Petukhova N 1999 Phys. Rev. B 591819

[19] Kera S, Yamane H, Sakuragi I, Okudaira K K, and Ueno N 2002 Chem. Phys. Lett. 36493 
[20] Lozzi L, Santucci S, La Rosa S, Delley B, and Picozzi S 2004 J. Chem. Phys. 1211883

[21] Yamane H, Yabuuchi Y, Fukagawa H, Kera S, Okudaira K K, and Ueno N 2006 J. Appl. Phys. 99093705

[22] Ellis T S, Park K T, Ulrich M D, Hulbert S L, and Rowe J E 2006 J. Appl. Phys. 100093515

[23] Downes J E, McGuinness C, Glans P-A, Learmonth T, Fu D, Sheridan P, and Smith K E 2004 Chem. Phys. Lett. 390203

[24] Rocco M, Frank K-H, Yannoulis P, and Koch E-E 1990 J. Chem. Phys. 936859

[25] Chambers D K, Karanam S, Qi D, Selmic S, Losovyj Ya B, Rosa L G, and Dowben P A 2005 Appl. Phys. A 80483

[26] Peisert H, Knupfer M, Schwieger T, Auerhammer J M, Golden M S, and Fink J 2002 J. Appl. Phys. 914872
[27] Hill I G, Makinen A J, and Kafafi Z H 2000 J. Appl. Phys. 88889

[28] Molotkov S N, Nazin S S, Smirnova I S, and Tatrskii V V 1991 Surf. Sci. 259339

[29] Tersoff J and Hamann D R 1985 Phys. Rev. B 31805

[30] Tersoff J 1990 Phys. Rev. B 411235

[31] Tersoff J and Hamann D R 1983 Phys. Rev. Lett. 501998

[32] Losovyj Ya B, Yakovkin I N, Barrett S D, Komesu T, and Dowben P A 2002 Surf. Sci. 52043

[33] Losovyj Ya, Zuber S M, and Dowben P A 2004 Vacuum 74 147

[34] Diehl R D and McGrath R 1997 J. Phys.: Condens. Matter 9 951 